\title{
How to change nurses' behavior leading to medication administration errors using a survey approach in United Christian Hospital
}

\author{
Lap Fung Tsang, Tak Kwan Yuk, So Yuen Alice Sham \\ Nursing Services Division, United Christian Hospital, Hospital Authority, Hong Kong, China
}

Correspondence: Tsang Lap Fung. Address: Room 250, 2/F, Block F, 130 Hip Wo Street, UCH, KLN, Kwun Tong, Hong Kong, China. Email: tsanlf1@ha.org.hk

Received: May 15, 2014

DOI : 10.5430/jnep.v4n12p17
Accepted: September 8, $2014 \quad$ Online Published: September 22, 2014

URL: http://dx.doi.org/10.5430/jnep.v4n12p17

\section{Abstract}

Background: Medication administrations errors (MAEs) occur frequently in the world. Preventive measures have been extensively studied, but there is very limited literature considering nurses' behavior leading to medication administration errors in hospitals.

Objectives: This study aimed to change nurses' behavior so as to reduce MAE rate. The objective of this study was to study the phenomenon of nurses' behavior during medication administration and to infer these behaviors that might cause MAE. The secondary outcome aimed to evaluate the effectiveness of existing preventive measures.

Methods: A convenience sampling design was employed to recruit around 1600 qualified nurses to participate in 6 identical surveying forums where a self-reported questionnaire was filled by participants under guidance led by the first author between August 2013 and September 2013.

Results: There is a significant decreasing trend of MAE from the peak at 0.61 to the current 0.22 per 1,000 occupied patient bed days after the surveying forums were organized. A variety of inappropriate behaviours of medication administration was identified. Most of them were found significantly associated with MAE. Length of time nurses have been working was thought to be an important factor to lead to MAE due to complacency and poor supervision. Other possible factors such as knowledge deficits, poor communication and poor speak-up culture were associated with MAE.

Conclusion: Risk of MAEs is inherent in medication administration, and if not properly managed, incidents will happen. Incidents will jeopardize nurses' work performance, influence the patient safety and sustainability of the relationship between nurses and patients. In this study, various risks of nurses' behaviour in medication administration have been revealed. The surveying forum might be a good way for nurses to self-evaluate their behavior and to perform proper ways of medication administration. Although there are different preventive measures implemented, information is not reached at nurses. The nursing implications were recommended to uphold safety of nursing behavior from personal to corporate level.

\section{Key words}

Nurses' behavior, Medication administration errors, Knowledge deficits, Complacency, Primary nursing, Preventive measures, Compliance 


\section{Background}

Medication administrations errors (MAEs) occur frequently in the world. Overall adult MAE rates were $5.6 \%$ to $35 \%$ of a total of non-IV opportunities for error and IV opportunities for error respectively ${ }^{[1]}$. Medication error has ranked amongst the second top risk areas and accounted for $19.8 \%$ in United Christian Hospital (UCH) as it could cause unnecessary harm to patients ${ }^{[2]}$. According to the summary report of the Institute for Safe Medication Practices (ISMP) Canada, medication administration and prescribing incidents were associated with death most frequently ${ }^{[3]}$.

Nurse spent one third of their time on medication-related activities ${ }^{[4]}$. They are subject to a range of practices and procedures, which are dictated through legal, management and medical requirements to ensure safe medication administration. Although there have many preventive measures been implemented, MAE still occurs. Human factor is the one accounting for cause of MAE. In one review study, human behavior adherence to implement medication administration is the area we need to study ${ }^{[5]}$. Another review studies also addressed on nurses' poor calculation competency, poor adherence to protocols, poor knowledge of medications and complacency those are acted as key causes in $\mathrm{MAE}^{[6,7]}$. It has been raised awareness about knowledge and behavior among nurses regarding medication safety. Indeed there is very limited literature considering change nurses' behavior to reduce medication incidents.

\section{Objectives}

This study aimed to change nurses' behavior so as to reduce MAE rate. The objective of this study was to study the phenomenon of nurses' behavior during medication administration and to infer these behaviors that might cause MAE. The secondary outcome aimed to evaluate the effectiveness of existing preventive measures.

\section{Methods}

\subsection{Sampling and setting}

The study was undertaken at a district hospital with total 1216 official beds in Hong Kong. About 1600 qualified nurses were invited to join the 6 identical surveying forums lasting for 30-minute using convenience sampling method between August 2013 and September 2013. In each surveying forum, subjects were guided to go through each part of the questionnaire. Detail of the questionnaire was explicitly described in the following text. All participants reported individually without any pressure because the first author could not know what they replied in the questionnaire during the forum. After completion of questionnaire, a 10-minute debriefing session was provided to update and clarify scenarios listed in the questionnaire. Nursing students and newly graduated nurses so-called preceptees were excluded because they had less working experience that might violate the result of this study. Only the enrolled and registered nurses at any rank were recruited regardless part-time nurses.

\subsection{Sample size}

We used a confidence interval of 6 and $52 \%$ of participants reflected their correct behavior on medication administration and knew the existing preventive measures. With the $95 \%$ confidence level and estimated population of nurses in hospital was about 1600, the sample size required should be about 230. Finally, there were 269 eligible participants selected for data analysis according to the conditions given.

\subsection{Development of scenario}

Case scenarios were generated from different medication administration incidents that were reported between September 2011 and September $2012^{[5]}$. The initial review of Tsang's study was to identify the contributory factors to errors in six domains of administration principles from the report of administration incidents of medication in $\mathrm{UCH}^{[5]}$. Administration errors were reviewed according to the report from the Quality and Safety Office. Factors contributing to administration 
errors were then identified and categorized into several domains. The various contributory factors to medication administration errors included five-right principles, communication, and allergy checking in the past year. A variety of contributory factors leading to administration error among six categories (known drug allergy, dangerous drugs, anti-coagulants, hypoglycemic agent, concentrated electrolytes, and other kinds of drug) were allocated to the six aforementioned domains associated with administration errors. Parts of scenarios were generated based on the six categories to indicate non-compliance, knowledge deficits, mal-practice and behavioral issues of medication administration. With reference to the incident report ${ }^{[5]}$, in addition, parts of scenarios were generated and discussed amongst the former General Manager (Nursing), clinical teaching coordinator and five clinical teachers.

The main theme of each scenario were as follows,

1) Behavior of checking electronic system with physician's prescription following patient admission;

2) Nursing compliance of drug reconciliation;

3) Nursing behavior on checking drug prescription sheets during medication administration;

4) Nursing behavior on checking patient identification;

5) Nursing behavior on managing unfamiliar, unclear medications and 'stat' drug;

6) Nursing behavior on checking 'high alert medication' and using intravenous infusion device; and

7) Nursing behavior on managing preoperative medications

Regarding the evaluation of existing preventive measures, it was essential to evaluate whether nurses know existing preventive measures. The main focus of this part were to evaluate if nurses,

1) understood contents of guidelines;

2) revealed report of medication incidents regularly;

3) thought there were enough guideline or policy;

4) participated medication safety sharing or forum;

5) eased to get information;

6) knew 'Drug Allergy Reference Card' and 'TALL man' labeling system; and

7) Knew there were sharing sessions organized by department and hospital.

\subsection{Data collections}

In each surveying session, participants were guided by the first author to answer one by one scenario to ensure that nurses reflected their real practice instead chose a correct answer. Only 8 designated departments including Accident \& Emergency (AED), Ear, Nose \& Throat (ENT), Orthopaedics \& Traumatology (O\&T), Obstetrics \& Gynaecology (O\&G), Paediatrics \& Adolescence Medicine (P\&AM), Medicines \& Geriatrics (M\&G), Psychiatrics (PSY) and Surgery (SURG) department were mainly recruited using convenience sampling design. This was because the scenarios were related to medication administration in in-patient setting. Other than 8 departments, nurses were also welcome to participate in the surveying forum. 274 questionnaires were distributed to participants in total.

\subsection{Ethical consideration}

Anonymity was assured throughout the study and the data collected were treated as confidential. All nurses were voluntarily to participate in the study without any punishment or incentives. Nurses who participated in the study forum indicated that they had given agreement without informed written consent required. However, an information sheet 
explained the details of the study and email notification were provided before the study and during the surveying forum. The study was approved by hospital general manager (nursing) who has rights to grant ethical approval in hospital.

\subsection{Questionnaire}

The study of Kim was adapted to develop the survey study ${ }^{[8]}$. The questionnaire included 4 parts: demographic data, nature of medication errors experienced by participants, scenario-based reflection and evaluation of existing preventive measures.

\subsection{Validation of the questionnaire}

A content validity of first draft of questionnaire was performed by four senior nursing officers (SNOs) in terms of design, contents and wordings. The questionnaire was translated into Chinese by a colleague who specializes in English translation after revision of the first draft. Three clinical teachers were invited to perform pre-tested to check the relevance, clarity and simplicity of questionnaire. Finally, face validity was performed by the second author to finalize the version of questionnaire.

\subsection{Data analysis}

The analysis included descriptive statistics, the Chi-square and correlation test to evaluate the data collected. Descriptive statistics was analyzed in expression of frequency, percentage and number by scarification of various departments, ranks, experiences and academic qualifications. Correlation analysis was used to test interdependence of existing preventive measures and MAE. Chi-square test was used to analyse the association between MAE and various nursing behaviors. All data analyses were conducted using the Statistical Package for Social Sciences version 19.0 (SPSS Inc., Chicago, IL). Data entry was performed by a trained statistical staff. The first author involved in the interpretation of findings.

\section{Results}

The enrolled and registered nurses covered in this study were nursing personnel registered in the register under the Nurses Registration Ordinance (Chapter 164) as at the survey date from August 20, 2013 to September 27, 2013. The number of enrolled and registered nurses covered was 274 . The characteristics of the respondents were listed in Table 1.

The response rate of the survey in eight departments overall was $26.4 \%$ (269 respondents out of 1020 nurses in 8 departments). Only 4 departments were found to have the response rate over twenty per cent. They were M\&G (30.0\%), O\&T (38.4\%), SURG (39.1\%) and O\&G (21.6\%). The response rate from other departments that was not over twenty per cent should be caution in the following analyses.

\subsection{I ncident rate of medication administration errors after the surveying forums}

There was a significant decreasing trend of MAE from the peak at 0.61 to the current 0.22 per 1,000 occupied patient bed days from September of 2013 to December 2013.

\subsection{Phenomenon of nurses' behaviour and its relationship of medication administration errors (MAEs)}

About $28.2 \%$ of nurses reported that they had committed 1 to 5 MAEs in the past year in their working unit, with the highest incidence in oral administration (18.2\%) followed by intravenous administration (8\%). Incidents were highly associated with wrong time (14.2\%) and dose $(9.1 \%) .8 .8 \%$ of nurses were found to commit MAE in the morning administration period and $6.6 \%$ in the evening period that were associated with manpower and workload. Working experience was, interestingly, significantly related to MAEs in committed and observed groups. 
The Clinical Management System (CMS) is one of the electronic systems for recording patient's medical information. Physician prescribed medication based on the CMS and nurses usually do counter check with the prescription. It was reported that eighty (29.74\%) nurses did not check the CMS after physician's prescription. Of these, prudently, 51 (42.5\%) nurses did not perform it in M\&G. On the other hand, it is crucial to check the system appropriately. It was evidenced that only $7.55 \%$ to $16.23 \%$ of nurses correctly checked the correct systems. The inappropriate checking on electronic system was found significant in wrong dosage incident in $M \& G(p<.02)$.

Table 1. Characteristics of respondents

\begin{tabular}{llll}
\hline \multirow{2}{*}{ Characteristics of nurses } & & Participants & \\
\cline { 3 - 4 } Rank & EN & Number (n) & Percentage (\%) \\
\hline \multirow{4}{*}{ RN } & 14 & 5.1 \\
& APN/NO & 222 & 82.5 \\
& WM & 30 & 11.1 \\
& AED & 2 & 0.7 \\
& ENT & 10 & 3.6 \\
Mepartment & M\&G & 1 & 0.4 \\
& O\&T & 122 & 44.5 \\
& PSY & 38 & 13.9 \\
& P\&AM & 10 & 3.6 \\
Nursing experience (yrs.) & SURG & 16 & 5.8 \\
& O\&G & 45 & 16.4 \\
& $<1$ & 27 & 9.9 \\
& $1-2$ & 18 & 6.6 \\
& $>2-4$ & 37 & 13.5 \\
& $>4-6$ & 35 & 12.8 \\
& $>6$ & 26 & 9.5 \\
Nursing Qualification & $<1$ & 157 & 57.3 \\
& $1-2$ & 42 & 15.3 \\
& $>2-4$ & 55 & 20.1 \\
& $>4-6$ & 38 & 13.9 \\
& $>6$ & 14 & 5.1 \\
& Diploma & 123 & 44.9 \\
& Bachelor degree & 40 & 14.6 \\
& Post-graduate Diploma & 167 & 60.9 \\
& Master degree or above & 13 & 4.7 \\
& & 51 & 18.6 \\
\hline \multirow{5}{*}{ Working experience in present unit (yrs.) } & &
\end{tabular}

In addition, it was reported that nurses who did not comply with drug reconciliation before medication administration were found to be highly associated with MAEs with regards to allergy $\left(\chi^{2}=8.9, d f=2, p=.012\right)$. Nurses who only read on medication administration record (MAR) (oral) sheet were significantly associated with wrong frequency in O\&T $\left(\chi^{2}=\right.$ $8.73, d f=1, p=.003)$. More experienced nurses who were failed to read through MAR sheets were significantly associated with MAE with regards to wrong frequency $\left(\chi^{2}=3.83, d f=1, p<.05\right)$. On the other hand, it was remarkable that $35.5 \%$ of nurses did not perform correct patient identification.

During the ' 5 rights' checking process, it was extraordinary behaviour for nurses who were not familiar with particular medications. Only $49.5 \%$ of nurses could take appropriate action to deal with unfamiliar medications. When there were 
some cases that the prescription was unclear, $36.9 \%$ of nurses did not take appropriate action to clarify ' 5 rights'. The situation was found frequently in length of time nurses having been working $\left(\chi^{2}=8.36, d f=1, p=.004\right)$, in turn, being significantly associated with MAE with regards to wrong frequency $\left(\chi^{2}=5.52, d f=1, p<.02\right)$.

When checking with 'high alert medication', $73.9 \%$ of nurses were found to perform inappropriate behaviour on counter checking. These behaviour were found significant risk of occurring MAEs such as wrong name of drug $\left(\chi^{2}=4.75, d f=1\right.$, $p<.03)$ and wrong route $\left(\chi^{2}=5.42, d f=1, p=.02\right)$ in M\&G. Further, when checking the parameter setting of infusion device, almost $37.5 \%$ of nurses could not perform appropriate checking behaviour. It was found that junior nurses might perform inappropriate behavior led to MAE $\left(\chi^{2}=9.0, d f=3, p<.03\right)$.

Physicians sometimes might prescribe 'stat' dose for patient under certain circumstances. When there was a question of medication administration regarding 'stat' medication, only $30.7 \%$ of nurses were to confirm the prescription with physician. Those who did not perform appropriate actions were found significant risk at wrong dosage $\left(\chi^{2}=4.55, d f=1, p\right.$ $<.04)$ in O\&T. Also, it was highly associated with length of time nurses having been working in $\operatorname{AED}\left(\chi^{2}=6.43, d f=2, p\right.$ $=.04)$.

In the study, it was very common to administer medication to patients who were not under their primary care in many departments and it was found to be highly risk of committing medication administration errors in the past 1 year in P\&AM $\left(\chi^{2}=9.74, d f=4, p<.05\right)$.

\subsection{Effectiveness of existing preventive measures}

One hundred and fifteen (42.0\%) nurses replied that sharing sessions of medication safety were held in their departments in recent 1 year and was highly associated with MAEs $\left(\chi^{2}=7.57, d f=2, p<.03\right) .185(67.5 \%)$ nurses replied they regularly read the report of MAEs disseminated by hospital. 90 (34.0\%) nurses knew the contents of the self-assessment guide for medication safety 2005. 182 (68.9\%) nurses knew the Drug Allergy Reference Card 2011 has been using as a reference. 77 (28.9\%) nurses knew the 'TALL man' lettering and color on labels for particular medications. $130(42.6 \%)$ nurses were aware of the sharing forum/ seminar regarding medication safety had been held in hospital in the past 1 year. 75 (28.1\%) nurses had participated in the sharing forum/ seminar regarding medication safety. It was highly associated MAE with the nursing awareness of the sharing forum/ $\operatorname{seminar}\left(\chi^{2}=4.55, d f=1, p<.04\right)$.

\section{Discussion}

This study has showed its creditability of actual prevalence of medication incident with reference to data of Advanced Incident Reporting System (AIRS) of Hong Kong Hospital Authority. It was supported with the highest incident in oral and intravenous medication administration. When comparing data between those committed MAEs and observed MAEs, however, the actual figures of committed incident were underestimated in this study because of self-reported by participants in this study.

Medication administration error could be caused by many factors that were identified as active failure or human factors at the sharp end of the health care process and latent failures or system deficiencies brought about by poor strategic decision ${ }^{[9,10]}$. Hambleton stated one or more latent failures lie dormant for a period of time until it intersects with an active failure to manifest in the form of an error ${ }^{[9]}$. Any behavior could cause another person to doubt their self-worth and threaten the judgment and skill execution of a nurse ${ }^{[1]}$. As an active failure is probably associated with human behavior, the study has been importantly identified various nurses' behaviour during the medication administration.

Non-compliance of medication administration is a complex process of behavior among nurses and related to many factors. In the aspect of medication safety, medication administration, storage and management of medications, and managing adverse events are regarded as crucial domains ${ }^{[12]}$. When nurses perform mal-behaviour on medication administration, it 
is said to be non-compliance, in turn, neglect of conduct, resulting in MAE probably. Therefore, studying nurse's behaviour of medication administration is very important. This study provided a platform for nurses to review their practices and behaviour according to various scenarios and to see how these inappropriate behaviours leading to MAE probably.

A literature reported that increasing workload could be more prone to medication errors ${ }^{[13]}$. In this study, length of time nurses have been working was highly associated with committing MAE. One of the reasons may be related to the nature of job roles as senior nurses need to supervise junior nurses apart from their extensive works. It also accounts for why nurses always or often administer oral and intravenous medication to patient that were not under their primary care. About $23.7 \%$ and $38.8 \%$ nurses often and always need to help other nurses to administer oral and intravenous medications to patients respectively who were not under their primary care.

Furthermore, most of medication incidents were found in the morning (0700-0800) and evening session (1600-1700) when lots of medications are required to administer. According to the definitions of the elements of primary nursing, nurses perform that daily assessments are patient-based but not based on tasks or geographic regions of the unit ${ }^{[14]}$. In this regard, it is difficult for nurses to adhere especially when some junior nurses take longer time to administer medications. Other nurses may choose administer medication to patient not under primary care. It was supported by the findings that about one-fourth nurses still could not follow the principle of primary nursing scheme. It could be accounted for the issues of manpower shortage, nursing workload and polypharmacy. Obviously, insufficient staffing and increasing workloads are the factors influencing accuracy of medication administration ${ }^{[15]}$. Managerial staff may consider adjusting in nursing routine because the two said sessions are the busiest period that needed to prepare extensive quantities of medications. In addition, a comparison of medications taken with the prescribed medications at patient admission has been made to ensure there are no duplications, omissions or interactions and that there is a need to continue current medications ${ }^{[16]}$.

Complacency and unwilling of speak-up amongst nurses were found in this study. It is very dangerous for nurses who think they know but in fact they do not know. It is because complacency of experienced nurses could lead to mistakes ${ }^{[17]}$. A study revealed that poor knowledge of medications and complacent behaviour of the nurses are the main reasons for MAEs ${ }^{[18]}$. The phenomenon of complacency was found commonly in experienced nurses. In some scenarios such as unfamiliar and unclear drugs, some of nurses did not clarify with physician but rather went on administering resulting in MAE. It would be a challenge to change one's mindset and need to address on in future.

Poor security of knowledge transfer from senior to junior was another problem found in this study. There is no systematic mechanism to monitor quality of supervision in units. Many training programmes were organized for junior nurses but seldom for senior nurses. Most of time senior nurses are required for clinical updates rather than junior nurses. Managerial nurses may consider a bit more system in the way that how senior nurses are supervised effectively.

In view of the current situation, information were transmitted through electronic mail but the fact is that few nurses know what updated guideline or other preventive measures were implemented. Some methods were found very effective to avoid MAE with evidently that formal incident sharing session can draw nurses' attention of medication safety ${ }^{[19]}$. Even though there were sharing sessions held in hospital, low attendance rate was noted. The findings should draw the attention of several bodies (e.g. quality and safety department and nursing services division) and managerial nurses. Indeed, 'app' is considered as a good communication way to disseminate message amongst nurses. Authorized party may consider in this area in future.

The strength of this study was to reflect nurses' behaviour of medication administration objectively under different situations. It is difficult to obtain factual information on nurses' behaviour of medication administration if using the other methods such as audit. This study has reported a variety of inappropriate nursing behaviours. Indeed, nurses are essential to comply with particular nursing guidelines and nursing standards which had been issued by organization and hospital when performing different procedures on medication administration. It would be questioned to many managerial staff why 
nurses performed these kinds of behaviour during medication administration. Several studies conducted to develop a systematic approach to medication error control but only focused on the revision of policies, procedures related to medication administration safety, other systematic improvement and using medication chart review and incident reports ${ }^{[20,21]}$. There is no study to point out what nurses actually behaved in the process of medication administration. This study could be regarded as good examples that might be referenced by other nurses in the world. Further study is required to explore the reason of not adhering to the guideline and standards.

The other concern should be how to reduce such behaviours amongst nurses. One of the strength of the study was to design the debriefing session after the forum so that the participants could clarify any unclear concept and update their knowledge immediately. Nevertheless, it could not change one's attitude on adherence of guideline and standards even though nurses obtained corrected information. Therefore, supervision becomes paramount in clinical setting in order to increase adhering to proper practice. It has been evidenced that ineffective nurse supervision when delegating administration caused medication administration errors ${ }^{[22,23]}$. Further study is required to explore how to perform effective supervision of nurses.

\subsection{Limitations}

Although the questionnaire has been covered with different scenarios, there are too many scenarios like drug omission were not explored in this study. The scenarios illustrated in this study were only based on information from incident report and suggestions from clinical teachers. Therefore, other scenarios that were not illustrated here should not be excluded.

Regarding the study design, it is unethical if observing nurses to give drugs in clinical areas and it is unrealistic to get real data from audit format. Therefore, we used a survey design including different scenarios based on previous MAEs to understand nursing behaviors. From the past experience, however, response rate was low if only sending survey invitation by email. Moreover, we hoped that nurses could reflect their real behaviors. But the fact is that it is difficult to ensure getting real reflection by email survey because they might choose appropriate answers. Therefore, nurses were invited to attend six identical surveying forums lasting for 30 minutes to ensure that each nurse should keep on a right track to reflect their real behavior according to different scenarios. In order to increase participation, different levels of managerial nurses were explained about the aim, design and methods of the study.

\subsection{Nursing implications}

This study revealed existing nurses' behaviour of medication administration. The findings have shown various extents of inappropriate behaviour practicing in the process of medication administrations that might be risk of incident. Some internal and external attributes were identified in this study such as knowledge deficits, inadequate speak-up culture, poor supervision and communication. The authors hope to draw attention of different parties like managerial person as well as of all nursing staff to minimize MAEs through the following recommendations.

1) Standardize and simplify medication checking system (E.g. IPMOE)

2) Foster speak-up culture

3) Regular training for nurses regardless length of time nurses have been working

4) Establish an effective supervision system

5) Explore effective communication way

6) Extension of resident pharmacist

7) Regular review on MAE and share learning point in any means

\section{Conclusion}

Risk of MAEs is inherent in medication administration, and if not properly managed, incidents will happen. Incidents will jeopardize nurses' work performance, influence the patient safety and sustainability of the relationship between nurses and 
patients. In this study, various risks of nurses' behaviour in medication administration have been identified. The surveying forum might be a good way for nurses to self-evaluate their behavior and to perform proper ways of medication administration. Although there are different preventive measures implemented, information is not reached at nurses. The nursing implications were recommended to uphold safety of nursing behavior from personal to corporate level. The findings also inspire further causing factors research and a continued multiple-level approach to the system- and policy-based resolution of potential risks for MAEs in healthcare settings.

\section{Acknowledgement}

We extend our sincere thanks to all nurses in United Christian Hospital for their participation in the study, and all Departmental Operation Managers (Nursing) and Ward Managers (Nursing) for their support in the study. We extend our much sincere thanks to Mr. William Poon T F (senior nursing officer) and Ms. Law S L (senior nursing officer) for their valuable opinion, and Ms. Chan Y M (Clinical Teaching Coordinator), her team members and Ms. Tung W C (Nursing School Teacher) to contribute in this study.

\section{Conflicts of interests}

None declared.

\section{References}

[1] McLeod MC, Barber N, Franklin BD. Methodological variations and their effects on reported medication administration error rates. Quality and Safety in Health Care. 2013; 22(4): 278-89.

[2] Leung LY. UCH Quality \& Safety Committee. Incidents trend \& SE/SUE reporting, 2013. Available from: http://uchweb5/qsd/Incident\%20Trend/UCH/2013/1st\%20Quarter.pdf.

[3] ISMP Canada Safety Bulletin. Deaths Associated with Medication Incidents: Learning from Collaborative Work with Provincial Oces of the Chief Coroner and Chief Medical Examiner. Institute for Safe Medication Practices Canada. 2013; $13(8)$ : 1-7.

[4] Keohane CA, Bane AD, Featherstone E, et al. Qualifying nursing workflow in medication administration. The Journal of Nursing Administration. 2008; 38(1): 19-26. PMid:18157001 http://dx.doi.org/10.1097/01.NNA.0000295628.87968.bc

[5] Tsang LF. Identify gaps between local and international measures to avoid administration error on 1-year review in United Christian Hospital, Hong Kong. Open Journal of Nursing. 2013; 3: 13-20. http://dx.doi.org/10.4236/ojn.2013.38A003

[6] Castledine G. Staff nurse removed from nursing register for mis-management of drug rounds. British Journal of Nursing. 2005; 14(18): 953. PMid:16237348 http://dx.doi.org/10.12968/bjon.2005.14.18.19879

[7] Preston RM. Drug errors and patient safety: the need for a change in practice. British Journal of Nursing. 2004; 13(2): 72-8. PMid:14997063 http://dx.doi.org/10.12968/bjon.2004.13.2.12037

[8] Kim KS, Kwon SH, Kim JA, et al. Nurses' perceptions of medication errors and their contributing factors in South Korea. Journal of Nursing Management. 2011; 19: 346-53. PMid:21507105 http://dx.doi.org/10.1111/j.1365-2834.2011.01249.x

[9] Hambleton M. Applying root cause analysis and failure mode and effect analysis to our compliance programs. J. Health Care Compliance. 2005; 7: 5.

[10] Reason J. Human error: models and management. BMJ. 2000; 320: 768-70. PMid:10720363

http://dx.doi.org/10.1136/bmj.320.7237.768

[11] Beyea SC. Intimidation in health care settings and patient safety. Patient Safety First. 2004; 80: 115-7.

[12] Johnson M, Tran DT, Young H. Developing risk management behaviors for nurses through medication incident analysis. International Journal of Nursing Practice. 2011; 17: 548-55. PMid:22103820 http://dx.doi.org/10.1111/j.1440-172X.2011.01977.x

[13] Hughes RG, Blegen MA. Medication administration safety. In R.G. Hughes. (Ed.), Patient safety and quality: An evidence-based handbook for nurses. U.S. Department of Health and Human Services, 2008. Available from:

http://www.ahrq.gov/professionals/clinicians-providers/resources/nursing/resources/nurseshdbk/nurseshdbk.pdf.

[14] Nursing Services Department Hospital Authority. 'Primary Nursing Implementation Guide', Hospital Authority, 2006.

[15] Copping C. Preventing and reporting drug administration errors. Nursing Times. 2005; 101(33): 32-4. PMid:16130498

[16] The Joint Commission. Comprehensive Accreditation Manual for Hospitals Update 2. Oakbrook Terrace, IL: Joint Commission Resources, Inc.; 2011.

[17] Parish C. Complacency to blame for transfusion mistakes. Nursing Standard. 2003; 17(45): 8. 
[18] Fanak F, Parham A, Mehrdad F, et al. Errors in preparation and administration of intravenous medications in the intensive care unit of a teaching hospital: An observational study. Australian College of Critical Care Nurses. 2008; 21: 110-16. PMid:18387813 http://dx.doi.org/10.1016/j.aucc.2007.10.004

[19] Waring J, Currie G, Crompton A, et al. An exploratory study of knowledge brokering in hospital settings: Facilitating knowledge sharing and learning for patient safety? Social Science \& Medicine. 2013; 98: 79-86. PMid:24331885 http://dx.doi.org/10.1016/j.socscimed.2013.08.037

[20] Armutlu M, Foley ML, Surette J, et al. Survey of nursing perceptions of medication administration practices, perceived sources of errors and reporting behaviours. Healthcare Quarterly (Toronto). 2008; 11: 58-65. http://dx.doi.org/10.12927/hcq.2008.19651

[21] Haw C, Stubbs J, Dickens G. An observational study of medication administration errors in old-age psychiatric inpatients. International Journal for Quality in Health Care. 2007; 19(4): 210-216. PMid:17562662 http://dx.doi.org/10.1093/intqhc/mzm019

[22] Hughes RG, Blegen MA. Medication Administration Safety. Patient Safety and Quality: An Evidence-Based Handbook for Nurses. 2008; 2: 397-457.

[23] Westbrook JI, Rob MI, Woods A, et al. Errors in the administration of intravenous medications in hospital and the role of correct procedures and nurse experience. BMJ Quality \& Safety. 2011; 20(12): 1027-34. PMid:21690248

http://dx.doi.org/10.1136/bmjqs-2011-000089 